Korean J. Math. 20 (2012), No. 1, pp. 125-135

\title{
ELLIPTIC PROBLEM WITH A VARIABLE COEFFICIENT AND A JUMPING SEMILINEAR TERM
}

\author{
Q-Heung Choi and Tacksun JunG*
}

\begin{abstract}
We obtain the multiple solutions for the fourth order elliptic problem with a variable coefficient and a jumping semilinear term. We have a result that there exist at least two solutions if the variable coefficient of the semilinear term crosses some number of the eigenvalues of the biharmonic eigenvalue problem. We obtain this multiplicity result by applying the Leray-Schauder degree theory.
\end{abstract}

\section{Introduction}

Let $\Omega$ be a bounded domain in $R^{n}$ with smooth boundary $\partial \Omega$. Let $c \in R$ and $b(x)$ be Hölder continuous in $\Omega$. In this paper we consider the multiplicity result for the following fourth order elliptic equation with the variable coefficient semilinear term and Dirichlet boundary condition

$$
\begin{gathered}
\Delta^{2} u+c \Delta u=b(x)\left((u+1)^{+}-1\right), \quad \text { in } \Omega, \\
u=0, \quad \Delta u=0 \quad \text { on } \partial \Omega,
\end{gathered}
$$

where $u^{+}=\max \{u, 0\}$ and $u^{-}=-\min \{u, 0\}$. Let $\lambda_{k}, k \geq 1$, denote the eigenvalues and $\phi_{k} k \geq 1$ the corresponding eigenfunctions, suitably normalized with respect to $L^{2}(\Omega)$ inner product, of the eigenvalue problem $\Delta u+\lambda u=0$ in $\Omega, u=0$ on $\partial \Omega$, where each eigenvalue $\lambda_{k}$ is repeated as often as its multiplicity. We recall that $\lambda_{1}<\lambda_{2} \leq \lambda_{3} \ldots \lambda_{n} \ldots \rightarrow+\infty$, and that $\phi_{1}(x)>0$ for $x \in \Omega$. The eigenvalue problem $\Delta^{2} u+c \Delta u=\mu u$ in $\Omega, u=0, \Delta u=0$ on $\partial \Omega$ has also infinitely many eigenvalues

Received February 1, 2012. Revised March 12, 2012. Accepted March 15, 2012. 2010 Mathematics Subject Classification: 35J30, 35J40.

Key words and phrases: Fourth order elliptic operator, variable coefficient semilinear term, Dirichlet boundary condition, Leray-Schauder degree.

This work (Choi) was supported by Basic Science Research Program through the National Research Foundation of Korea (NRF) funded by the Ministry of Education, Science and Technology (KRF-2011-0003191).

${ }^{*}$ Corresponding author. 
$\mu_{k}=\lambda_{k}\left(\lambda_{k}-c\right), k \geq 1$ and corresponding eigenfunctions $\phi_{k}, k \geq 1$. We note that

$$
\lambda_{1}\left(\lambda_{1}-c\right)<\lambda_{2}\left(\lambda_{2}-c\right) \leq \lambda_{3}\left(\lambda_{3}-c\right)<\cdots .
$$

Tarantello [9] studied problem (1.1) with $b(x)=b$ constant;

$$
\begin{aligned}
& \Delta^{2} u+c \Delta u=b\left((u+1)^{+}-1\right), \\
& u=0, \quad \Delta u=0 \quad \text { on } \partial \Omega .
\end{aligned}
$$

She showed that if $c<\lambda_{1}$ and $b \geq \lambda_{1}\left(\lambda_{1}-c\right)$, then (1.2) has at least two solutions, one of which is a negative solution. She obtained this result by the degree theory. Micheletti, Pistoia and Saccon [8] also proved that if $c<\lambda_{1}$ and $b \geq \lambda_{2}\left(\lambda_{2}-c\right)$, then (1.2) has at least three solutions by the variational linking theorem and Leray-Schauder degree theory. Choi and Jung [2] showed that the problem

$$
\begin{array}{ll}
\Delta^{2} u+c \Delta u=b u^{+}+s & \text { in } \Omega, \\
u=0, \quad \Delta u=0 \quad \text { on } \partial \Omega
\end{array}
$$

has at least two nontrivial solutions when $c<\lambda_{1}, \lambda_{1}\left(\lambda_{1}-c\right)<b<$ $\lambda_{2}\left(\lambda_{2}-c\right)$ and $s<0$ or when $\lambda_{1}<c<\lambda_{2}, b<\lambda_{1}\left(\lambda_{1}-c\right)$ and $s>0$. They obtained these results by using the variational reduction method. They [3] also proved that when $c<\lambda_{1}, \lambda_{1}\left(\lambda_{1}-c\right)<b<\lambda_{2}\left(\lambda_{2}-c\right)$ and $s<0,(1.3)$ has at least three nontrivial solutions by using degree theory. They [4] also proved that the single fourth order elliptic problem with some nonlinearity has at least three solutions. In $[4,5,6,9]$ the authors investigate the existence of multiple solutions of fourth order elliptic problems.

In this paper we improve these results to the problem (1.1) with the variable coefficient jumping semilinear term $b(x)$. Now we consider the eigenvalue problem

$$
\begin{aligned}
& \Delta^{2} u+c \Delta u-b(x) u=\Lambda u \quad \text { in } \Omega, \\
& u=0, \quad \Delta u=0 \quad \text { on } \partial \Omega .
\end{aligned}
$$

McKenna and Walter [7] showed that (1.4) has infinitely many eigenvalues $\Lambda_{k}, k \geq 1$, and the corresponding eigenfunctions $\psi_{k}, k \geq 1$. We assume that the eigenfunctions are normalized with respect to $H$ inner product (the space $H$ is introduced in section 2). Standard eigenvalue theory gives that

$$
\Lambda_{1}<\Lambda_{2} \leq \Lambda_{3} \leq \cdots, \quad \Lambda_{k} \rightarrow+\infty \quad \text { as } k \rightarrow+\infty,
$$




$$
\psi_{1}(x)>0 \quad \text { in } \Omega .
$$

Our main results are as follows:

THEOREM 1.1. Let $c<\lambda_{1}, \lambda_{n}\left(\lambda_{n}-c\right)<b(x)<\lambda_{n+1}\left(\lambda_{n+1}-c\right)$, $n \geq 1$. Then (1.1) has at least two nontrivial solutions, one of which is a negative solution.

We use the Leray-Schauder degree theory to prove main results. The outline of the proofs is as follows: In section 2, we show the existence of the negative solution and investigate the a priori bound for the solutions of (1.1) under the assumptions of Theorem 1.1 and prove Theorem 1.1.

\section{The Hilbert space and proof of Theorem 1.1}

Any element $u$ in $L^{2}(\Omega)$ can be written as

$$
u=\sum h_{k} \psi_{k} \quad \text { with } \sum h_{k}^{2}<\infty .
$$

We define a subspace $H$ of $L^{2}(\Omega)$ as follows

$$
H=\left\{u \in L^{2}(\Omega)\left|\sum\right| \Lambda_{k} \mid h_{k}^{2}<\infty\right\} .
$$

Then this is a complete normed space with a norm

$$
\|u\|=\left[\sum\left|\Lambda_{k}\right| h_{k}^{2}\right]^{\frac{1}{2}} .
$$

Since $\Lambda_{k} \rightarrow+\infty$ and $c$ is fixed, we have

(i) $\left(\Delta^{2}+c \Delta-b(x)\right) u \in H$ implies $u \in H$.

(ii) $\|u\| \geq C\|u\|_{L^{2}(\Omega)}$, for some $C>0$.

(iii) $\|u\|_{L^{2}(\Omega)}=0$ if and only if $\|u\|=0$.

For the proof of the above results we refer [1].

Lemma 2.1. Assume that $c$ is not an eigenvalue of $-\Delta, \Lambda_{k} \neq 0$ (or $\left.b(x) \neq \lambda_{k}\left(\lambda_{k}-c\right)\right)$, and $b(x)$ is bounded. Then all solutions in $L^{2}(\Omega)$ of

$$
\Delta^{2} u+c \Delta u=b(x)\left((u+1)^{+}-1\right) \quad \text { in } \quad L^{2}(\Omega)
$$

belong to $H$.

Proof. (1.1) can be rewritten as

$$
\begin{array}{ll}
\left(\Delta^{2}+c \Delta-b(x)\right) u=b(x)(u+1)^{-} & \text {in } \Omega, \\
u=0, \quad \Delta u=0, & \text { on } \partial \Omega
\end{array}
$$


Let us write $b(x)(u+1)^{-}=\sum h_{k} \psi_{k} \in L^{2}(\Omega)$.

$$
\begin{aligned}
\left(\Delta^{2}+c \Delta-b(x)\right)^{-1} b(x)(u+1)^{-} & =\sum \frac{1}{\Lambda_{k}} h_{k} \psi_{k} \in L^{2}(\Omega) . \\
\mid\left(\Delta^{2}+c \Delta-b(x)\right)^{-1} b(x)(u+1)^{-} \| & =\sum\left|\Lambda_{k}\right| \frac{1}{\left(\Lambda_{k}\right)^{2}} h_{k}^{2} \\
& \leq C \sum h_{k}^{2}=C\|u\|_{L^{2}(\Omega)}^{2}<\infty
\end{aligned}
$$

for some $C>0$. Thus $\left(\Delta^{2}+c \Delta-b(x)\right)^{-1} b(x)(u+1)^{-} \in H$.

With the aid of Lemma 2.1 it is enough to show that we investigate the existence of the solutions of (1.1) in the subspace $H$ of $L^{2}(\Omega)$.

Now we shall calculate the Leray-Schauder degree on the neighborhood of a unique negative solution $u^{*}$ of the linear problem

$$
\begin{array}{cc}
\Delta^{2} u+c \Delta u=-b(x), & \text { in } \Omega, \\
u=0, \quad \Delta u=0 \quad \text { on } \partial \Omega
\end{array}
$$

Lemma 2.2. Assume that $c<\lambda_{1}, \lambda_{n}\left(\lambda_{n}-c\right)<b(x)<\lambda_{n+1}\left(\lambda_{n+1}-c\right)$, $n \geq 1$. Then there exists a constant $\eta>0$ such that the Leray-Schauder degree

$$
d_{L S}\left(u-\left(\Delta^{2}+c \Delta\right)^{-1}\left(b(x)\left((u+1)^{+}-1\right)\right), B_{\eta}\left(u^{*}\right), 0\right)=(-1)^{n} .
$$

Proof. We know that the linear problem (2.4) has a unique solution $u^{*}$. Since $c<\lambda_{1}$ and $-b(x)<0$, by applying the standard strong maximum principle to $z=\Delta u$ and consequently to $u, u^{*}$ is negative. Let $u$ be a nontrivial solution of (1.1). Since $b(x)\left((u+1)^{+}-1\right) \geq-b(x)$, by the standard strong maximum principle to $z=\Delta u$ and consequently to $u$, we have $u>u^{*}$. Since $\min \left\{(u+1)^{+}-1\right\}=-1$ and $b(x)>\lambda_{1}\left(\lambda_{1}-c\right)$,

$$
\min _{\Omega} u<-1 \text {, }
$$

so

$$
\|u\|_{L^{\infty}}>1 \text {. }
$$

By the Schauder estimates for elliptic operator, $\left(\Delta^{2}+c \Delta\right)^{-1}$ is a linear compact operator from $H$ into $H$. We note that if $u \in H,(u+1)^{+}-1 \in$ $H$. Thus the operator $u-\left(\Delta^{2}+c \Delta\right)^{-1}\left(b(x)\left((u+1)^{+}-1\right)\right)$ is well defined from $H$ into $H$. (1.1) can be rewritten as

$$
\left(\Delta^{2}+c \Delta-b(x)\right) u=b(x)(u+1)^{-} .
$$


or

$$
u=\left(\Delta^{2}+c \Delta-b(x)\right)^{-1}\left(b(x)(u+1)^{-}\right) .
$$

Let $K$ be the closure of $\left(\Delta^{2}+c \Delta-b(x)\right)^{-1}(\bar{B})$, where $\bar{B}$ is the closed unit ball centered at $u^{*}$ in $L_{2}(\Omega)$. Let $u$ be a nontrivial solution of (1.1). Let $u=u^{*}+v$ and $\|v\|=\eta$. Then $v$ satisfies the equation

$$
\left(\Delta^{2}+c \Delta-b(x)\right) v=b(x)\left(u^{*}+v+1\right)^{-}+b(x) u^{*}+b(x)
$$

or

$$
v=\left(\Delta^{2}+c \Delta-b(x)\right)^{-1}\left(b(x)\left(u^{*}+v+1\right)^{-}+b(x) u^{*}+b(x)\right) .
$$

Let us set $\beta=\max b(x)$. Since $\left\|\left(u^{*}+v+1\right)^{-}\right\|<\left\|\left(u^{*}+v\right)^{-}\right\|$and

$$
\begin{aligned}
\left\|b(x)\left(u^{*}+v+1\right)^{-}+b(x) u^{*}+b(x)\right\| & \leq\left\|b(x)\left(u^{*}+v\right)^{-}+b(x) u^{*}+b(x)\right\| \\
& \leq \beta\left(\eta+2\left\|u^{*}\right\|+1\right) .
\end{aligned}
$$

Thus

$$
v \in \beta(\eta+1) K
$$

From (2.9) we get

$$
\begin{aligned}
v+\left(\Delta^{2}\right. & +c \Delta-b(x))^{-1}\left(-b(x) u^{*}-b(x)\right) \\
& =\left(\Delta^{2}+c \Delta-b(x)\right)^{-1}\left(b(x)\left(u^{*}+v+1\right)^{-}\right) .
\end{aligned}
$$

Let us set $y=\frac{v}{\eta}$. Then $\|y\|=1$ and $y \in \beta\left(1+\frac{1}{\eta}\right) K$. Since $y$ is in compact set and different from 0 and since $b(x)>0$ is not eigenvalue, we can choose a number $\eta>0$ so small that

$$
\begin{aligned}
\inf _{y} \| y & +\frac{1}{\eta}\left(\Delta^{2}+c \Delta-b(x)\right)^{-1}\left(-b(x)-b(x) u^{*}\right) \| \\
& \geq \inf _{y}\left\|y+\frac{1}{\eta}\left(\Delta^{2}+c \Delta-b(x)\right)^{-1}(-b(x))\right\|=a>0
\end{aligned}
$$

since $\left(\Delta^{2}+c \Delta\right)^{-1}\left(-b(x)-b(x) u^{*}\right)>\left(\Delta^{2}+c \Delta\right)^{-1}(-b(x))$ and $\|\left(\Delta^{2}+\right.$ $c \Delta-b(x))^{-1}(-b(x)) \|=\frac{\beta}{\min \left\{b(x)-\lambda_{n}\left(\lambda_{n}-c\right), \lambda_{n+1}\left(\lambda_{n+1}-c\right)-b(x)\right\}}>1$. Hence the norm of the left hand side of $(2.10)$ is

$$
\left\|v+\left(\Delta^{2}+c \Delta-b(x)\right)^{-1}\left(-b(x)-b(x) u^{*}\right)\right\|>a \eta .
$$

On the other hand, we shall estimate the norm of the right hand side of (2.10). If $v$ is a solution of (2.8), then by $\left\|\left(\Delta^{2}+c \Delta-b(x)\right)^{-1}\right\|=$ 
$\frac{1}{\min \left\{b(x)-\lambda_{n}\left(\lambda_{n}-c\right), \lambda_{n+1}\left(\lambda_{n+1}-c\right)-b(x)\right\}}$, the norm of the right hand side of (2.10) is

$$
\begin{aligned}
\|\left(\Delta^{2}+c \Delta\right. & -b(x))^{-1}\left(b(x)\left(u^{*}+v+1\right)^{-}\right) \| \\
& \leq \frac{\beta}{\min \left\{b(x)-\lambda_{n}\left(\lambda_{n}-c\right), \lambda_{n+1}\left(\lambda_{n+1}-c\right)-b(x)\right\}} \eta .
\end{aligned}
$$

We note that $\frac{\beta}{\min \left\{b(x)-\lambda_{n}\left(\lambda_{n}-c\right), \lambda_{n+1}\left(\lambda_{n+1}-c\right)-b(x)\right\}}>1$. Now we choose $\eta>0$ so small that $\frac{\beta}{\min \left\{b(x)-\lambda_{n}\left(\lambda_{n}-c\right), \lambda_{n+1}\left(\lambda_{n+1}-c\right)-b(x)\right\}} \eta<a \eta$. Thus for this value of $\eta$, there is no solution of (1.1) of the form $u=u^{*}+v$ with $\|v\|=\eta$. That is,

$$
u-\left(\Delta^{2}+c \Delta\right)^{-1}\left(b(x)(u+1)^{+}-b(x)\right) \neq 0 \quad \text { on } \partial B_{\eta}\left(u^{*}\right) .
$$

We apply the similar argument to the equation

$$
\begin{aligned}
& \left(\Delta^{2}+c \Delta-b(x)\right) u \\
& =\lambda b(x)(u+1)^{-}+\lambda b(x) u^{*}+\lambda b(x)-b(x)-b(x) u^{*} \quad \text { in } H,
\end{aligned}
$$

where $0 \leq \lambda \leq 1$ and $u^{*}$ is the unique negative solution of (2.4). $\lambda=1$ gives the equation (1.1). Let $u$ be a nontrivial solution of (2.11) for arbitrary $\lambda$ with $0 \leq \lambda \leq 1$. Let $u=u^{*}+v$. Then $v$ satisfies the equation

$$
\left(\Delta^{2}+c \Delta-b(x)\right)(v)=\lambda b(x)\left(u^{*}+v+1\right)^{-}+\lambda b(x) u^{*}+\lambda b(x)
$$

or

$$
\text { (2.13) } v=\left(\Delta^{2}+c \Delta-b(x)\right)^{-1}\left(\lambda b(x)\left(u^{*}+v+1\right)^{-}+\lambda b(x) u^{*}+\lambda b(x)\right) \text {. }
$$

Let $u=u^{*}+v$ and $\|v\|=\eta$. Let us set $\beta=\max b(x)$. Since $\|\left(u^{*}+v+\right.$ $1)^{-}\|<\|\left(u^{*}+v\right)^{-} \|$and

$$
\begin{aligned}
& \left\|\lambda b(x)\left(u^{*}+v+1\right)^{-}+\lambda b(x) u^{*}+\lambda b(x)\right\| \\
& \leq\left\|b(x)\left(u^{*}+v\right)^{-}\right\|+\left\|b(x) u^{*}\right\|+\|b(x)\| \\
& \leq \beta\left(\eta+2\left\|u^{*}\right\|+1\right) .
\end{aligned}
$$

Thus

$$
v \in \beta(\eta+1) K .
$$

From (2.13) we get

$$
\begin{aligned}
v+\left(\Delta^{2}\right. & +c \Delta-b(x))^{-1}\left(-\lambda b(x) u^{*}-\lambda b(x)\right) \\
& =\left(\Delta^{2}+c \Delta-b(x)\right)^{-1}\left(\lambda b(x)\left(u^{*}+v+1\right)^{-}\right) .
\end{aligned}
$$


Elliptic problem with a variable coefficient and a jumping semilinear term 131

By the same argument as the previous discussion, there is no solution of (1.1) of the form $u=u^{*}+v$ with $\|v\|=\eta$. That is,

$$
\begin{aligned}
& u-\left(\Delta^{2}+c \Delta-b(x)\right)^{-1}\left(\lambda b(x)(u+1)^{-}+\lambda b(x) u^{*}+\lambda b(x)-b(x)-b(x) u^{*}\right) \\
& \neq 0 \quad \text { on } \partial B_{\eta}\left(u^{*}\right) .
\end{aligned}
$$

Since the Leray-Schauder degree is invariant under a homotopy, we have

$$
\begin{aligned}
& d_{L S}\left(u-\left(\Delta^{2}+c \Delta\right)^{-1}\left(b(x)(u+1)^{+}-b(x)\right), B_{\eta}\left(u^{*}\right), 0\right) \\
& =d_{L S}\left(u-\left(\Delta^{2}+c \Delta-b(x)\right)^{-1}\left(\lambda b(x)(u+1)^{-}\right.\right. \\
& \left.\left.\quad \quad+\lambda b(x) u^{*}+\lambda b(x)-b(x)-b(x) u^{*}\right), B_{\eta}\left(u^{*}\right), 0\right) \\
& \left.=d_{L S}\left(u-\left(\Delta^{2}+c \Delta-b(x)\right)^{-1}\left(-b(x)-b(x) u^{*}\right)\right), B_{\eta}\left(u^{*}\right), 0\right) \\
& =d_{L S}\left(u-\left(\Delta^{2}+c \Delta\right)^{-1}(b(x) u), B_{\eta}(0), 0\right) .
\end{aligned}
$$

Now we are trying to find the number of the negative eigenvalues of the equation

$$
u-\left(\Delta^{2}+c \Delta\right)^{-1}(b(x) u)=\sigma u .
$$

We note that $u-\left(\Delta^{2}+c \Delta\right)^{-1}(b(x) u)=\sigma u$ is equivalent to the equation

$$
\left(\Delta^{2}+c \Delta\right) u-r b(x) u=0, \text { where } r=\frac{1}{1-\sigma}
$$

and $\sigma<0$ corresponds to $0<r<1$. We first consider the eigenvalue problem

$$
\left(\Delta^{2}+c \Delta\right) u-r \lambda_{n}\left(\lambda_{n}-c\right) \frac{b(x)}{\lambda_{n}\left(\lambda_{n}-c\right)} u=0 .
$$

Since $\frac{b(x)}{\lambda_{n}\left(\lambda_{n}-c\right)}>1, r_{k}\left(\lambda_{n}\left(\lambda_{n}-c\right)\right)<\lambda_{k}\left(\lambda_{k}-c\right)$. Thus

$$
r_{k}<\frac{\lambda_{k}\left(\lambda_{k}-c\right)}{\lambda_{n}\left(\lambda_{n}-c\right)}
$$

We next consider the eigenvalue problem

$$
\left(\Delta^{2}+c \Delta\right) u-r \lambda_{n+1}\left(\lambda_{n+1}-c\right) \frac{b(x)}{\lambda_{n+1}\left(\lambda_{n+1}-c\right)} u=0 .
$$

Since $\frac{b(x)}{\lambda_{n+1}\left(\lambda_{n+1}-c\right)}<1, r_{k}\left(\lambda_{n+1}\left(\lambda_{n+1}-c\right)\right)>\lambda_{k}\left(\lambda_{k}-c\right)$. Thus

$$
\frac{\lambda_{k}\left(\lambda_{k}-c\right)}{\lambda_{n+1}\left(\lambda_{n+1}-c\right)}<r_{k}
$$


By (2.17) and (2.18),

$$
\frac{\lambda_{k}\left(\lambda_{k}-c\right)}{\lambda_{n+1}\left(\lambda_{n+1}-c\right)}<r_{k}<\frac{\lambda_{k}\left(\lambda_{k}-c\right)}{\lambda_{n}\left(\lambda_{n}-c\right)} .
$$

Thus there exist $n$ numbers of $r_{k}, k=1,2, \cdots, n$ in the area of $0<r_{k}<$ 1 , so there exist $n$ number of negative eigenvalues $\sigma$ of (2.16). Thus we have

$$
d_{L S}\left(u-\left(\Delta^{2}+c \Delta\right)^{-1}(b(x) u), B_{\eta}(0), 0\right)=(-1)^{n},
$$

so we prove the lemma.

Lemma 2.3. Assume that $c<\lambda_{1}$ and $\lambda_{n}\left(\lambda_{n}-c\right)<b(x)<\lambda_{n+1}\left(\lambda_{n+1}-\right.$ $c)$. Then there exists a constant $s_{0}>0$ so large enough that if $s \geq s_{0}$, then the problem

$$
\begin{aligned}
& \Delta^{2} u+c \Delta u=b(x)\left((u+1)^{+}-1\right)+s \psi_{1}(x), \quad \text { in } \Omega, \\
& u=0, \quad \Delta u=0 \quad \text { on } \partial \Omega
\end{aligned}
$$

has no solution.

Proof. We suppose that the lemma is false. Then there exist a sequence $u_{n}$ in $H$ and $\left(s_{n}\right)_{n \in N}$ such that $\lim _{n \rightarrow \infty} s_{n}=+\infty$ and $u_{n}$ are the solutions of

$$
\begin{aligned}
& u_{n}=\left(\Delta^{2}+c \Delta\right)^{-1}\left(b(x)\left(\left(u_{n}+1\right)^{+}-1\right)+s_{n} \psi_{1}(x)\right), \quad \text { in } \Omega, \\
& u_{n}=0, \quad \Delta u_{n}=0 \quad \text { on } \partial \Omega .
\end{aligned}
$$

We claim that $\left\{u_{n}\right\}$ is unbounded. In fact, if $\left\{u_{n}\right\}$ is bounded, then $\lim _{n \rightarrow \infty} \frac{u_{n}}{s_{n}}=0$ in $\Omega$, strongly in $L^{2}(\Omega)$ and weakly in $H$. Dividing (2.20) by $s_{n}$, we have

$$
\frac{u_{n}}{s_{n}}=\left(\Delta^{2}+c \Delta\right)^{-1}\left(b(x)\left(\left(\frac{u_{n}}{s_{n}}+\frac{1}{s_{n}}\right)^{+}-\frac{1}{s_{n}}\right)+\psi_{1}(x)\right) .
$$

Passing to the limit to both sides of (2.21), we have that

$$
0=\left(\Delta^{2}+c \Delta\right)^{-1}\left(\psi_{1}(x)\right),
$$

which is a contradiction because the left hand side of the equality is 0 , but the right hand side is not equal to 0 . Thus $\lim _{n \rightarrow \infty}\left\|u_{n}\right\|=\infty$. Let $z_{n}=\frac{u_{n}}{\left\|u_{n}\right\|}$. Since $\left\{z_{n}\right\}$ is compact, there exists a subsequence, up to a subsequence, $\left\{z_{n}\right\}$ such that $\lim _{n \in N} z_{n}=z$ a.e. in $\Omega$, strongly in $L^{2}(\Omega)$ and weakly in $H$. Dividing (2.20) by $\left\|u_{n}\right\|$, we have

$$
z_{n}=\left(\Delta^{2}+c \Delta\right)^{-1}\left(b(x)\left(\left(z_{n}+\frac{1}{\left\|u_{n}\right\|}\right)^{+}-\frac{1}{\left\|u_{n}\right\|}\right)+\frac{s_{n}}{\left\|u_{n}\right\|} \psi_{1}(x)\right) .
$$


We suppose that $\lim _{n \rightarrow \infty} \frac{s_{n}}{\left\|u_{n}\right\|}=s \geq 0$. Passing to the limit in (2.22), we obtain that

$$
\begin{aligned}
& z=\left(\Delta^{2}+c \Delta\right)^{-1}\left(b(x) z^{+}+s \psi_{1}(x)\right) . \\
& z=0, \quad \Delta z=0 \quad \text { on } \partial \Omega .
\end{aligned}
$$

We claim that (2.23) has only trivial solution $z=0$. In fact, we suppose that $z \neq 0$. Multiplying both sides of $(2.23)$ by $\psi_{1}(x)$ and integrating, we have

$$
0 \leq s=\int_{\Omega}\left(\left(\Delta^{2}+c \Delta\right) z \psi_{1}(x)-b(x) z^{+} \psi_{1}(x)\right) d x,
$$

which is absurd because the right hand side of the above equation is negative since $\Lambda_{1}<0$ and $-\left(\Lambda_{1}+b(x)\right)<0$ (which come from the assumption $c<\lambda_{1}$ and $\lambda_{n}\left(\lambda_{n}-c\right)<b(x)<\lambda_{n+1}\left(\lambda_{n+1}-c\right)$. Thus $z=0$. This is a contradiction since $\|z\|=1$. We prove the lemma.

Lemma 2.4. (A priori bound) Assume that $c<\lambda_{1}$ and $\lambda_{n}\left(\lambda_{n}-c\right)<$ $\left.b(x)<\lambda_{n+1}\left(\lambda_{n+1}-c\right)\right)$. If there exist a constant $C>0$ and $s_{*}>0$ with $s_{*}<s_{0}$ such that all solutions $u$ of (2.19) with $s \leq s_{*}$, then $\|u\| \leq C$.

Proof. We argue by contradiction. Suppose that there exists a sequence $\left(u_{n}, s_{n}\right)$ such that $\left\|u_{n}\right\| \rightarrow \infty, s_{n} \rightarrow s_{*}, s_{n} \leq s_{*}$ and $u_{n}$ satisfy the equation

$$
\begin{aligned}
& \left(\Delta^{2}+c \Delta-b(x)-\Lambda_{1}\right) u_{n} \\
& \quad=-\Lambda_{1} u_{n}^{+}+\Lambda_{1} u_{n}^{-}+b(x)\left(u_{n}+1\right)^{-}+s_{n} \psi_{1}(x) .
\end{aligned}
$$

Let $z_{n}=\frac{w_{n}}{\left\|w_{n}\right\|}$. By the compactness of $\left\{z_{n}\right\}$, there exists $z$ such that $z_{n} \rightarrow z$ and $z$ is a solution of the equation

$$
\left(\Delta^{2}+c \Delta-b(x)-\Lambda_{1}\right) z=-\Lambda_{1} z^{+}+\Lambda_{1} z^{-}+b(x) z^{-} .
$$

Taking inner product of both sides of $(2.25)$ with $\psi_{1}(x)$, we have

$$
\begin{aligned}
0 & =\left(\left(\Delta^{2}+c \Delta-b(x)-\Lambda_{1}\right) z, \psi_{1}(x)\right) \\
& =\left(-\Lambda_{1} z^{+}+\left(\Lambda_{1}+b(x)\right) z^{-}, \psi_{1}(x)\right) .
\end{aligned}
$$

Since $-\Lambda_{1} z^{+}+\left(\Lambda_{1}+b(x)\right) z^{-} \geq \epsilon\|z\|$, the right hand side of (2.26) is bigger than or equal to 0 . Thus the only possibility to hold (2.26) is that $z=0$, which is impossible since $\|z\|=1$. Thus we prove the lemma. 
Lemma 2.5. Assume that $c<\lambda_{1}, \lambda_{n}\left(\lambda_{n}-c\right)<b(x)<\lambda_{n+1}\left(\lambda_{n+1}-c\right)$, $n \geq 1$. Then there exists a constant $R>0$ (depending on $C$ which is introduced in Lemma 2.4) such that any solutions of (1.1) are contained in $B_{R}(0)$ and the Leray-Schauder degree

$$
d_{L S}\left(u-\left(\Delta^{2}+c \Delta\right)^{-1}\left(b(x)(u+1)^{+}-b(x)\right), B_{R}(0), 0\right)=0
$$

for $R>C$.

Proof. This result follows from Lemma 2.3 and Lemma 2.4. By Lemma 2.3 , there exists a constant $s_{0}>0$ such that if $s \geq s_{0}$, (2.19) has no solution. By Lemma 2.4, there exist a constant $C$ and $s_{*}>0$ with $s_{*}<s_{0}$ such that if $u$ is a solution of (2.19) with $s<s_{*}$, then $\|u\| \leq C$. Let us choose $R$ so large that $R>C$. We note that $u-\left(\Delta^{2}+c \Delta\right)^{-1}\left(b(x)(u+1)^{+}-b(x)+(1-\lambda) s_{0} \psi_{1}(x)\right)=u-\left(\Delta^{2}+\right.$ $\left.c \Delta-b(x)-\Lambda_{1}\right)^{-1}\left(-\Lambda_{1} u^{+}+\Lambda_{1} u^{-}+b(x)(u+1)^{-}+(1-\lambda) s_{0} \psi_{1}(x)\right) \neq 0$ on $\partial B_{R}(0)$ for $0 \leq \lambda \leq 1$. By the homotopy invariance property, we have that the Leray-Schauder degree

$$
\begin{aligned}
& d_{L S}\left(u-\left(\Delta^{2}+c \Delta\right)^{-1}\left(b(x)(u+1)^{+}-b(x)\right), B_{R}(0), 0\right) \\
& =d_{L S}\left(u-\left(\Delta^{2}+c \Delta\right)^{-1}\left(b(x)(u+1)^{+}-b(x)+(1-\lambda) s_{0} \psi_{1}(x)\right), B_{R}(0), 0\right) \\
& =d_{L S}\left(u-\left(\Delta^{2}+c \Delta\right)^{-1}\left(b(x)(u+1)^{+}-b(x)+s_{0} \psi_{1}(x)\right), B_{R}(0), 0\right)=0,
\end{aligned}
$$

where $0 \leq \lambda \leq 1$. Thus we prove the lemma.

\section{Proof of Theorem 1.1}

By Lemma 2.5, there exists a large number $R>0$ (depending on $C$ ) such that the Leray-Schauder degree

$$
d_{L S}\left(u-\left(\Delta^{2}+c \Delta\right)^{-1}\left(b(x)(u+1)^{+}-b(x)\right), B_{R}(0), 0\right)=0
$$

for $R>C$. By Lemma 2.2, there exists a constant $\eta>0$ such that the Leray-Schauder degree

$$
d_{L S}\left(u-\left(\Delta^{2}+c \Delta\right)^{-1}\left(b(x)(u+1)^{+}-b(x)\right), B_{\eta}\left(u^{*}\right), 0\right)=(-1)^{n},
$$

where $u^{*}$ is a unique negative solution of the linear problem

$$
\begin{aligned}
& \Delta^{2} u+c \Delta u=-b(x), \quad \text { in } \Omega, \\
& u=0, \quad \Delta u=0 \quad \text { on } \partial \Omega .
\end{aligned}
$$

If $n$ is even, then the Leray-Schauder degree in the region $B_{R}(0) \backslash B_{\eta}\left(u^{*}\right)$ is -1 , so there exists the second solution of (1.1) in the region $B_{R}(0) \backslash B_{\eta}\left(u^{*}\right)$. Therefore there exist at least two solutions of (1.1), one of which is a negative solution. If $n$ is odd, then the Leray-Schauder degree in the 
Elliptic problem with a variable coefficient and a jumping semilinear term 135

region $B_{R}(0) \backslash B_{\eta}\left(u^{*}\right)$ is +1 , so there exists the second solution of (1.1) in the region $B_{R}(0) \backslash B_{\eta}\left(u^{*}\right)$. Therefore there exist at least two solutions of (1.1), one of which is a negative solution. Thus we complete the proof.

\section{References}

[1] Q.H. Choi and T.Jung, Multiplicity of solutions and source terms in a fourth order nonlinear elliptic equation, Acta Math. Sci. 19 (4) (1999), 361-374.

[2] Q.H. Choi and T.Jung, Multiplicity results on nonlinear biharmonic operator, Rocky Mountain J. Math. 29, (1) (1999), 141-164.

[3] T.S. Jung and bf Q.H. Choi, Multiplicity results on a nonlinear biharmonic equation, Nonlinear Anal. 30 (8) (1997), 5083-5092.

[4] T.Jung and Q.H. Choi, On the existence of the third solution of the nonlinear biharmonic equation with Dirichlet boundary condition, Chungcheong Math. J., 20 (2007), 81-94.

[5] A.C. Lazer and P.J. McKenna, Multiplicity results for a class of semilinear elliptic and parabolic boundary value problems, J. Math. Anal. Appl., 107 (1985), 371-395.

[6] P.J. McKenna and W. Walter, On the multiplicity of the solution set of some nonlinear boundary value problems, Nonlinear Anal. 8 (1984), 893-907.

[7] P.J. McKenna and W. Walter, On the multiplicity of the solution set of some nonlinear boundary value problems-II, Nonlinear Anal. 10 (8) (1986), 805-812.

[8] A.M. Micheletti, A. Pistoia and C. Saccon, Three solutions of a 4 th order elliptic problem via variational theorems of mixed type, Appl. Anal. 75 (2000), 43-59.

[9] Tarantello, A note on a semilinear elliptic problem, Differential Integral Equations, 5 (3) (1992), 561-565.

Department of Mathematics Education

Inha University

Incheon 402-751, Korea

E-mail: qheung@inha.ac.kr

Department of Mathematics

Kunsan National University

Kunsan 573-701, Korea

E-mail: tsjung@kunsan.ac.kr 